\title{
A global attractivity result for a class of switching discrete-time systems
}

\author{
Selim Solmaz ${ }^{*}$, Robert Shorten, and Fiacre Ó Cairbre
}

\begin{abstract}
In this paper we present the global attractivity properties of a class of discrete-time switching systems of the form $x(k+1)=A_{i} x(k)$, $A_{i} \in \mathscr{A} \triangleq\left\{A_{1}, \ldots, A_{m}\right\}$, where each constituent matrices $A_{i} \in \mathbb{R}^{n \times n}$ are Schur stable. We assume that a set of non-singular matrices $T_{i j} \in \mathbb{R}^{n \times n}$ exist such that the matrices $T_{i j} A_{i} T_{i j}^{-1}$ and $T_{i j} A_{j} T_{i j}^{-1}$ for $i, j \in\{1, \ldots, m\}$ are upper triangular. We show that for a special subset of such switching systems the origin is globally attractive, and it is possible to prove this without requiring the existence of a common quadratic Lyapunov function (CQLF).
\end{abstract}

\section{INTRODUCTION}

It is known that the existence of a common quadratic Lyapunov function (CQLF) is sufficient to guarantee the exponential stability of the discrete time switching system

$$
x(k+1)=A_{i} x(k), A_{i} \in \mathscr{A},
$$

where $\mathscr{A} \triangleq\left\{A_{1}, \ldots, A_{m}\right\}$ with Schur stable constituent matrices $A_{i} \in$ $\mathbb{R}^{n \times n}$ for $i \in\{1, \ldots, m\}$, and $x(k) \in \mathbb{R}^{n}$. A sufficient condition for the existence of a CQLF, $V(x)=x^{T} P x, P=P^{T}>0, \quad P \in \mathbb{R}^{n \times n}$ for (1) is that a non-singular transformation $T$ exists such that $T A_{i} T^{-1}$ is upper triangular for all $i \in\{1, \ldots, m\}$ [4]. This result was first derived in [1] for continuous time switched systems, and further discussed in [2] and [3]. Unfortunately, from a practical viewpoint, the requirement of simultaneous triangularizability imposes unrealistic conditions on the matrices in $\mathscr{A}$. Relaxation of this requirement for continuous time switching systems has been analyzed in [5], [6], [7] extensively, where they did not specifically require the existence of a CQLF for the switched Hurwitz stable systems. It was assumed, amongst other conditions, that any pair of matrices in $\mathscr{A}$ are pairwise triangularizable. In general pairwise triangularizability is not sufficient for the existence of a CQLF for the switched systems, yet it was shown using a non-Lyapunov approach in [5] that the origin of a special subclass of pairwise triangularizable switching continuous-time systems is globally attractive. In this paper we investigate the discrete-time analog of these results.

A traditional approach to relate continuous-time linear time invariant (LTI) Hurwitz stability results to discrete-time LTI Schur stability counterparts require the use of bilinear transform. However as reported in a recent paper [8], it is not straightforward to use the bilinear transform to relate continuous-time and discretetime switching systems. It therefore follows that the stability of a special subclass of pairwise triangularizable switching discrete-time systems do not necessarily follow from continuous-time systems with this property, and that the stability of this system class must be investigated using a 'first principles approach'.

\section{MAIN RESUlT}

We first give the formal definition of pairwise triangularizability which we will refer in the rest of the paper.

S. Solmaz (selim.solmaz@nuim.ie) and R. Shorten (robert.shorten@nuim.ie) are both with the Hamilton Institute, National University of Ireland-Maynooth, Co. Kildare, Ireland. Fiacre Ó Cairbre (Fiacre.ocairbre@maths.nuim.ie) is with the Department of Mathematics, National University of Ireland-Maynooth, Co. Kildare Ireland

${ }^{*}$ Corresponding author. Phone:+353 1 7086100, Fax: +353 17086269
Definition (Pairwise Triangularizability): Let a switching system described by (1) be given. Suppose that a number of non-singular matrices $T_{i j}$ exist, such that for each pair of matrices $\left\{A_{i}, A_{j}\right\}$ in $\mathscr{A}$, where $i, j \in\{1, \ldots, m\}$ and $i \neq j$, the pair of matrices $\left\{T_{i j} A_{i} T_{i j}^{-1}, T_{i j} A_{j} T_{i j}^{-1}\right\}$ are upper triangular. Then every distinct pair of matrices $\left\{A_{i}, A_{j}\right\}$ in $\mathscr{A}$ are called pairwise triangularizable.

While the ultimate objective of the work is to determine the global attractivity and stability of the origin of (1) (where any two $A_{i}$ matrices can be simultaneously triangularized), for the purpose of this paper we consider simpler systems where, amongst other conditions, the $A_{i}$ matrices in $\mathscr{A}$ are diagonalizable, and where any two of the $A_{i}$ matrices have at least $n-1$ real linearly independent eigenvectors in common. In this case, the origin of the switching system is globally attractive as verified in the following theorem.

Theorem 2.1: Let $\mathscr{V}=\left\{v_{1}, \ldots, v_{n+1}\right\}$ be a set of real vectors, where each $v_{i} \in \mathbb{R}^{n}$ for $i=\{1,2, \ldots, n+1\}$. Suppose any $n$ vectors in $\mathscr{V}$ are linearly independent. For each $i \in\{1,2, \ldots, n+1\}$, we construct $M_{i} \in \mathbb{R}^{n \times n}$ matrices as follows

$$
M_{i}=\left\{\begin{array}{ccc}
{\left[v_{1}, \ldots, v_{i-1}, v_{i}, \ldots, v_{n}\right]} & \text { for } & i=1 \\
{\left[v_{1}, \ldots, v_{n+1}, v_{i}, \ldots, v_{n}\right]} & \text { for } & 2 \leq i \leq n+1
\end{array}\right.
$$

i.e., $M_{i}$ is obtained by replacing the $(i-1)^{t h}$ column in $M_{1}$ with the vector $v_{n+1}$. Suppose we also have $p$ different diagonal matrices $D_{1}, D_{2}, \ldots, D_{p}$ in $\mathbb{R}^{n \times n}$ with all diagonal entries in the right half of the unit circle, i.e., for every diagonal entry $\lambda_{h, j}$ of $D_{h}$, we can write

$$
0<\lambda_{h, j}<1, \quad \text { for } \quad 1 \leq h \leq p, \quad 1 \leq j \leq n .
$$

We now define the matrices $A_{h, i} \in \mathbb{R}^{n \times n}$ as follows

$$
A_{h, i}=M_{i} D_{h} M_{i}^{-1},
$$

and let $\mathscr{A}$ be the set of all $A_{h, i}$ for $h \in\{1,2, \ldots, p\}$ and $i \in$ $\{1,2, \ldots, n+1\}$. Then for the switching system (1) with the set $\mathscr{A}$ defined as above, the origin is globally attractive.

Comment 2.1: The following facts can be deduced for the set $\mathscr{A}$ defined in Theorem 2.1:

(i) Every matrix in $\mathscr{A}$ is Schur stable and diagonalizable.

(ii) Any matrix pair in $\mathscr{A}$ share at least $(n-1)$ linearly independent common real eigenvectors.

(iii) Every matrix pair in $\mathscr{A}$ can simultaneously be triangularized. (See [4] for the proof of this.)

Proof of Theorem 2.1: The line of proof is similar to the continuous time version given in [5] (see the proof of Theorem 3.1 in [5]), and here we only present an outline of the main ideas of the proof.

Step 1 : We replace each $n \times n$ matrix $M_{j}$ by an $(n+1) \times(n+1)$ matrix $\bar{M}_{j}$. We then replace each $n \times n$ matrix $A_{h, i}$ in $\mathscr{A}$ by an $(n+1) \times(n+1)$ matrix $\bar{A}_{h, i}$. The matrices $\bar{A}_{h, i} \in \overline{\mathscr{A}} \triangleq\left\{\bar{A}_{h, i}: A_{h, i} \in\right.$ $\mathscr{A}\}$ are chosen such that there is at least one common eigenvector $\tau=(1,0,0, \ldots, 0)$ for all the matrices in $\overline{\mathscr{A}}$, and also such that the properties of the solutions of the dynamic system

$$
\bar{x}(k+1)=\bar{A}(k) \bar{x}(k), \bar{A}(k) \in \overline{\mathscr{A}},
$$

will ultimately imply the global attractivity of the origin of the system (1), where $x=\left(x_{1}, \ldots, x_{n}\right)$ and $\bar{x}=\left(x_{n+1}, x_{1}, \ldots, x_{n}\right)$. 
Step 2 : For a given $j \in\{1,2, \ldots, n+1\}$ we consider the $n+1$ linearly independent columns of $\bar{M}_{j}$. These form an $n+1$ dimensional coordinate system which includes $\tau$ as one of the axes. We consider the projection of the state $\bar{x}(k)$ onto $\tau$ as the dynamics of the system (5) evolve. This projection is given by the first component of the vector

$$
g_{j}(k)=\bar{M}_{j}^{-1} \bar{x}(k),
$$

and is denoted by $\left[g_{j}\right]_{1}(k)$.

Step 3: We then show that $\lim _{k \rightarrow \infty}\left|\left[g_{j}\right]_{1}(k)-\left[g_{i}\right]_{1}(k)\right|=$ $0, \overline{\forall i, j} \in\{1, \ldots, n+1\}$. From this fact we can deduce that $\lim _{k \rightarrow \infty}\left(x_{1}, \ldots, x_{n}\right)=0$. This is sufficient to demonstrate the global attractivity of the origin of the system (1).

Comment 2.2: We can not simply replace Hurwitz stable matrices for the continuous-time case in Theorem 3.1 of [5] with Schur stable matrices and arrive at the same conclusions of global attractivity of the origin. In the discrete-time case we need the condition given in equation (3) on the eigenvalues of $D_{h}$ for $1 \leq h \leq$ $p$. Because otherwise, we do not get the global asymptotic stability of the origin. This is demonstrated in the following example.

Example 2.1: Let the set $\mathscr{V}=\left\{v_{1}, v_{2}, v_{3}, v_{4}\right\}$ be given as

$$
\begin{array}{ll}
v_{1}=\left[\begin{array}{lll}
1 & 0 & 0
\end{array}\right]^{T}, & v_{2}=\left[\begin{array}{lll}
0 & 1 & 0
\end{array}\right]^{T}, \\
v_{3}=\left[\begin{array}{lll}
0 & 0 & 1
\end{array}\right]^{T}, & v_{4}=\left[\begin{array}{lll}
1 & 1 & 1
\end{array}\right]^{T} .
\end{array}
$$

Further assume that $M_{i} \in \mathbb{R}^{3 \times 3}$ matrices are constructed as follows

$$
\begin{array}{rll}
M_{1} & =\left[\begin{array}{lll}
v_{1} & v_{2} & v_{3}
\end{array}\right], & M_{2}=\left[\begin{array}{lll}
v_{4} & v_{2} & v_{3}
\end{array}\right], \\
M_{3}=\left[\begin{array}{lll}
v_{1} & v_{4} & v_{3}
\end{array}\right], & M_{4}=\left[\begin{array}{lll}
v_{1} & v_{2} & v_{4}
\end{array}\right] .
\end{array}
$$

Moreover select a $3 \times 3$ diagonal Schur stable matrix $D$ as follows

$$
D=\left[\begin{array}{ccc}
0.9 & 0 & 0 \\
0 & 0.8 & 0 \\
0 & 0 & -0.6
\end{array}\right]
$$

Now consider the following Schur stable LTI systems

$$
\Sigma_{A_{i}}: x(k+1)=A_{i} x(k), A_{i} \in \mathbb{R}^{3 \times 3},
$$

where $A_{i}$ matrices are constructed from

$$
A_{i}=M_{i} D M_{i}^{-1}, \quad i=1, . ., 4 .
$$

It is sufficient to show that there exists a switching sequence between $A_{i}$ 's such that the resulting system has eigenvalues outside the unit circle. We simply consider the incremental switching sequence $A_{1} \rightarrow A_{2} \rightarrow A_{3} \rightarrow A_{4}$; then the dynamics of the system evolve according to the matrix product

$$
A=A_{1} A_{2} A_{3} A_{4} .
$$

Since the eigenvalues of $A$ are $\{1.1899,0.1058,0.2766\}$, then with one eigenvalue outside the unit circle, this switching sequence is unstable. It is also interesting to note that if $D$ is chosen such that all of the eigenvalues are on the right half of the unit circle, i.e., $D=\operatorname{diag}\{0.9,0.8,0.6\}$, then the $A$ matrix corresponding to the switching sequence (9) has eigenvalues $\{0.5861,0.1517,0.3917\}$ and is stable by Theorem 2.1.

\section{CONCLUDING REMARKS}

In this paper we have shown that the global attractivity results for a class of discrete-time switching systems is not necessarily equivalent to continuous time systems with this property. Hence, in cases when the existence of a CQLF is unknown for the switched set of LTI systems, qualitative statements concerning the system stability for the continuous-time as well as discrete-time systems must be validated separately using non-CQLF techniques. One such technique is presented in this paper; namely, a technique which proves global attractivity by embedding the original ( $n$-dimensional) state space in a higher $(n+1)$ dimensional state space. Finally, we note that our motivation for this study was to solve an automotive control stabilization problem that is related to vehicle rollover, and which involves switching. Future work will report the (successful) application of the results in this paper to this problem.

\section{Acknowledgements}

This work was partially supported by Science Foundation Ireland Grant 04/IN3/I478.

\section{REFERENCES}

[1] Y. Mori, T. Mori, and Y. Kuroe, "A Solution to the Common Lyapunov Function Problem for Continuous Time Systems," in proceedings of 36th Conference on Decision and Control, 1997.

[2] D. Liberzon, J. P. Hespanha, and S. Morse, "Stability of Switched Linear Systems: A Lie Algebraic Condition," tech. rep., Laboratory for Control Science and Engineering, Yale University, 1998.

[3] R. Shorten and K. Narendra, "On the Stability and Existence of Common Lyapunov Functions for Linear Stable Switching Systems." In proceedings of Conference on Decision and Control, Florida Dec 15th-18th, 1998.

[4] R. Shorten, A Study of Hybrid Dynamical Systems with Application to Automotive Control. $\mathrm{PhD}$ thesis, Department of Electrical and Electronic Engineering, University College Dublin, Republic of Ireland, 1996.

[5] R. Shorten and F. Ó Cairbre, "A Proof of Global Attractivity for a Class of Switching Systems using a non-Lyapunov Approach.” IMA Journal of Mathematical Control and Information (2001) 18, 341-353.

[6] R. Shorten and F. Ó Cairbre, "On the Stability of Pairwise Triangularisable and Related Switching Systems." Proceedings of the American Control Conference (2001) 3, 1882 - 1883.

[7] R. Shorten and F. Ó Cairbre, "A New Methodology for the Stability Analysis of Pairwise Triangularizable and Related Switching Systems." IMA Journal of Applied Mathematics (2002) 67, 441-457.

[8] T. Mori, T.V. Nguyen, Y. Mori, and H. Kokame, "Preservation of Lyapunov Functions under Bilinear Mapping," Automatica (2006) 42, 1055-1058. 